\title{
Gefangen zwischen Besserung und Sicherung - Ein Plädoyer für die regelmäßige Prüfung der Gefährlichkeit in der Hauptverhandlung
}

\author{
Gönke Jacobsen
}

$\mathrm{n}$ einem von Sicherheits- und Sicherungsbestrebungen gekennzeichneten kriminalpolitischen Klima beansprucht seit geraumer Zeit die betroffene Praxis eindringlich Gehör. Strafvollzugsempiriker und Psychowissenschaftler schlagen ebenfalls Alarm: Nicht nur der Strafvollzug, sondern auch der (psychiatrische) Maßregelvollzug leiden erheblich unter konstanter Überbelegung. Neben der kriminologischen Diskussion um die Gründe für die aktuelle Überbelegung (zum Strafvollzug Dünkel/Geng; zur Situation des Maßregelvollzuges Dessecker 2005; Royen; Jehle) werden bei der Auswahl und Vollstreckung von freiheitsentziehenden Sanktionen praktische Fragen relevant. Wie wirkt sich die Überbelegungssituation auf die ministerielle Steuerung bei der Gewährung von Vollzugslockerungen und die Aussetzung des Strafrestes durch die Strafvollstreckungskammern aus? Welche Rolle spielt überhaupt noch der Besserungsauftrag des Strafvollzuges gemäß $\S 2$ Satz 1 StVollzG, der als Vollzugsziel den Normenkanon des Gesetzes anführt? Im Folgenden soll zu der auf Boetticher (1998, S. 354 ff) zurückgehenden Diskussion Stellung genommen werden, ob die regelmäßige Prüfung der Gefährlichkeit in der Hauptverhandlung erfolgen sollte, damit das vorhersehbare Dilemma bei der Strafrestaussetzung besser vorbereitet werden kann.

\section{Die Angst vor dem medial konstruier- ten, extrem gefährlichen, meist sexuell motivierten Serientäter.}

Durch die veränderte Wahrnehmung der Gefahren, die von Gewalt- und Sexualstraftätern ausgehen, muss die Kriminalpolitik in Deutschland den Eindruck vermitteln, "alles im Griff" zu haben. Die seit dem Jahr 1998 erlassenen Gesetze zur Bekämpfung von Sexualdelikten und anderen gefährlichen Straftaten, die mit der Einführung der nachträglichen Sicherungsverwahrung ihren vorläufigem Höhepunkt erreicht haben, hatten deshalb zum Ziel, das Sicherheitsgefühl der Bevölkerung nachhaltig zu stärken. Einzelne Ermittlungserfolge durch
DNA-Identifizierung von Sexualtätern runden die scheinbar erfolgreiche Politik ab.

Die Angst der Bürger, die zu dieser Sicherheitsgesetzgebung geführt hat, ist auf den medial konstruierten, extrem gefährlichen, meist sexuell motivierten Serientäter fokussiert. Hat die Bundesregierung ihr Ziel erreicht? Ist es jetzt sicherer als vorher? Dies sind Fragen, die Politiker sich stellen, wenn als Betrachtungsgruppe der schuldfähige, rückfallgefährdete, also gefährliche Gewaltstraftäter gewählt wird. Ihre Antwort darauf ist nur vordergründig einfach: Es sollte im Urteil Sicherungsverwahrung nach den $\S \S 66$ ff. StGB angeordnet werden. Unter den Voraussetzungen des $§ 66$ Abs. 1 ist die Verhängung der Sicherungsverwahrung obligatorisch, wenn der Täter bereits zwei Anlasstaten begangen hat, dafür mindestens 2 Jahre Freiheitsstrafe verbüßt hat und wiederum zu Freiheitsstrafe von mindestens 2 Jahren verurteilt wird. Absatz 3 hingegen eröffnet dem Gericht hinsichtlich der Anordnung der Maßregel bei näher bezeichneten Gewaltstraftaten Ermessen, bei einer Verurteilung zu mindestens 3 Jahren Freiheitsstrafe sogar ohne vorherige Anlasstaten. Eine Unterbringung in einem psychiatrischen Krankenhaus gem. § 63 StGB kommt bei der genannten Tätergruppe allerdings nicht in Betracht, da die Schwelle des $\S \S 20,21$ StGB nicht erreicht ist oder die geforderte Kausalität zwischen psychischem Defekt und der Tat nicht besteht. Um das Problem zu erfassen, muss die Betrachtungsgruppe somit erneut verengt werden: Es geht stets um den schuldfähigen, rückfallgefährdeten, also gefährlichen Gewaltstraftäter, der zu einer zeitigen Freiheitsstrafe ohne anschließende Sicherungsverwahrung verurteilt wurde. Dabei ist jedoch nicht ausgeschlossen, dass eine therapierbare, psychische Störung unterhalb der Anforderungen der §§ 20, 21 StGB vorliegt, die gerade die Gefährlichkeit begründen oder steigern kann. Freese beziffert immerhin den Anteil von psychisch kranken Straftätern im Strafvollzug mit einer Zahl zwischen 10-30 \% (S. 52). Bennefeld-Kersten (S. 38) ermittelte eine Anzahl von 2,6\% der Stichtagsbelegung für den Strafvollzug in Niedersachsen, die aufgrund psychischer Auffälligkeiten einer gesonderten Unterbringung bedurft hätten. Da mit sehr engen Auswahlkriterien gearbeitet wurde, geht sie davon aus, dass der Anteil an Gefangenen, die generell an einer psychischen Beeinträchtigung leiden, um ein Vielfaches höher liege. Der Gang des Verfahrens ist auch hier eindeutig: Ist das Gefährdungspotential des Täters bereits in der Hauptverhandlung erkannt worden oder zumindest erkennbar - dies stellt den Regelfall dar -, so gelangt der Täter nach mehreren Jahren in die Freiheit zurück und fällt nicht in den Anwendungsbereich der nachträglichen Sicherungsverwahrung (vgl. BGH v. 11.5.2005, StV 2005, S. 388 ff.; OLG Koblenz v. 21.09.2004, NStZ 2005, 97, das betont hat, dass eine gescheiterte Resozialisierung keine neue Tatsache iSd $\S 66 \mathrm{~b}$ StGB ist).

\section{Die Gefährlichkeitsprüfung bei der Strafrestaussetzung - eine Sackgasse?}

Um dem Sicherheitsbedürfnis der Bürger in diesen Fällen dennoch genüge $\mathrm{zu}$ tun, muss die Strafvollstreckungskammer gem. § 454 Abs. 2 StPO gutachterlichen Rat über die Gefährlichkeit des (Gewalt-) Täters einholen, bevor der Strafrest der Freiheitsstrafe zur Bewährung ausgesetzt wird (zur Frage, in welchen Fällen von der Hinzuziehung eines Sachverständigen abgesehen werden kann, Neubacher). Nur wenn während des Vollzuges neue Erkenntnisse über den Täter gewonnen werden (bspw. das Begehen weiterer Taten im Vollzug, vgl. BT-Drs. 15/2887, S. 12), die die Gefährlichkeit des Täters begründen oder steigern, so kann in einem zweiten Schritt das Landgericht (§ 74f GVG) erst unter diesen Umständen eine nachträgliche Sicherungsverwahrung nach $\S 66 \mathrm{~b}$ StGB anordnen.

Die genannte Straftätergruppe wird nicht nur in den tagespolitischen Medien als hochgefährlich dargestellt. Auch $\S 66$ Abs. 3 StGB indiziert dieses Attribut und bei der Strafrestaussetzung, also im Vollstreckungsverfahren, stehen die Verurteilten ebenfalls unter dem Generalver- 
dacht der Gefährlichkeit. In der Hauptverhandlung hingegen geht es um den Kampf um die Schuldfähigkeit und um das Umgehen der Thematik der Sicherungsverwahrung. Elz hat in einer Untersuchung von Verfahren gegen Sexualstraftäter herausgefunden, dass nur in etwa einem Viertel der untersuchten Urteile ein Sachverständiger bestellt wurde, um den Täter auf psychische Störungen hin zu untersuchen (S. 153 ff.). Mehrheitlich hätte es sich dabei um Schuldfähigkeitsbegutachtungen gehandelt, nur ein nicht näher bezeichneter Teil enthielte Ausführungen über die Gefährlichkeit der Täter, was sie auf die Vorgaben des Begutachtungsauftrages zurückführt. Das bedeutet, dass in 75 \% der Fälle das erkennende Gericht die Einschaltung eines professionellen Gutachters nicht für nötig befunden hat. Weiter stellt Elz fest, dass sich immerhin in jeweils einem Drittel der Urteile Ausführungen über die Schuldfähigkeit und die Gefährlichkeit der Angeklagten finden (S. 172 ff). Dabei wurde in maximal $28 \%$ aller Urteile eine verminderte Schuldfähigkeit angenommen, in maximal $15 \%$ eine Gefährlichkeit des Täters erkannt. Baltzer hat eine Untersuchung von Gewaltstraftätern im hessischen Strafvollzug vorgelegt, die zu zeitiger Freiheitsstrafe ohne weitere Maßregelanordnung verurteilt wurden. Er kommt zu dem Ergebnis, dass in 52,6\% der Fälle ein psychiatrischpsychologischer Sachverständiger hinzugezogen wurde (S. 158). Zu einer von ihm gebildeten Hochrisikogruppe (18\% der Gesamtstichprobe) stellt er fest, dass nur in wenigen dieser Fälle sich die erkennenden Gerichte mit der Frage der Anordnung von Sicherungsverwahrung befasst haben (S. 161). Es wird anhand dieser kleinen Datenschau deutlich, dass bei einer Tätergruppe, die im Vollstreckungsverfahren im Hinblick auf die Gefährlichkeit grundsätzlich begutachtet werden muss, dieser Aspekt im Hauptverfahren vernachlässigt wird.

Nedopil als psychiatrischer Sachverständiger kritisiert im Zusammenhang mit der Strafrestaussetzung im Strafvollzug, dass der Beratungsspielraum des Gutachters für die Gerichte gegen Null gehe (S. 349). Würden Lockerungen ausbleiben, würden Gutachter eine günstige Entlassungsprognose nicht begründen können; Gerichte könnten ohne entsprechendes Gutachten eine vorzeitige Entlassung nicht anordnen. Die Hypothese der Gefährlichkeit, so Nedopil, bliebe bis zur Verbüßung der Endstrafe nicht entkräftet und die Entlassungsprognose bliebe weiterhin ungünstig. Dies wiederum hätte nahezu zwangsläufig die Einweisung in eine Anschlussunterbringung zur Folge, wobei sich die Justiz auf Kosten der Psychiatrie und deren Gutachter entlastet hätte.

In Bezug auf die Freilassung dieser Täter befürchtet Nedopil eine Verantwortungsverlagerung von den Gerichten hin zu den zuständigen Sachverständigen im Rahmen der Strafvollstreckung. Das Argument des Bundesverfas- sungsgerichts zur Begründung der sog. Schwurgerichtslösung bei § 57a StGB zur Feststellung der Schwere der Schuld gilt entsprechend auch in diesem Zusammenhang: Das Grundrecht auf ein faires, rechtsstaatliches Verfahren gebiete es, die Feststellungen zur Schuldschwere dem erkennenden Gericht zu übertragen. Nach Auffassung des Bundesverfassungsgerichts könne wegen des großen zeitlichen Abstandes zwischen der Tat und seiner Entscheidung das Vollstreckungsgericht diesen Rechtsstaatlichen Garantien nicht mehr genügen (BVerfGE 86, 288, 317 ff.; zur Auswirkung dieser Rechtsprechung Speiermann, S. 68 ff.). Die Frage, ob ein Täter überhaupt gefährlich ist, ist zwar für die Vollstreckung der Freiheitsstrafe relevant, deren Beantwortung am Ende der Vollstreckung allerdings erscheint wenig hilfreich. Es bleibt festzuhalten, dass der Zeitpunkt am Ende der Strafvollstreckung in jedem Fall zu spät ist.

Es ist deshalb aus dieser Sicht konsequent, dass derzeit gefordert wird, die Frage der Gefährlichkeit nicht am Ende der Strafvollstreckung zu klären, sondern stets zum Gegenstand der Hauptverhandlung zu machen. Eine interdisziplinäre Arbeitsgruppe beim Bundesgerichtshof stellt fest: »Häufig werden (...) die Sachverständigen über die Angaben zur Schuldfähigkeit hinaus nicht zur Gefährlichkeitsprognose und zu den Behandlungsaussichten in der sozialtherapeutischen Anstalt befragt, obwohl sachverständige Angaben zu Therapiemöglichkeiten und deren Auswirkungen auf die Rückfallwahrscheinlichkeit sinnvoll sind, damit im Vollstreckungsverfahren schon früh die Weichen für eine adäquate Behandlung des Beschuldigten gesteckt werden. Dies sollte über die Beachtung des § 246a StPO hinaus zum Standard jeder Hauptverhandlung gehören und sich im Strafurteil, das Grundlage für das Vollstreckungsverfahren ist, wieder finden « (Boetticher/Nedopil/Bosinski/Saß, S. 59).

\section{Inhalt des Begriffs »gefährlicher Gewaltstraftäter«}

Es ist zu betonen, dass es bei dieser Debatte um Täter geht, die Delikte der in $\S 66$ Abs. 3 StGB genannten Art begangen haben und die damit per se als gefährlich erachteten werden. Die Gruppe umfasst alle Verbrechen (insb. Raub, Mord, Vergewaltigung) sowie explizit aufgeführte Vergehen wie die Missbrauchstatbestände mit Kindesbezug und die gefährliche Körperverletzung. Damit stimmt diese Deliktsgruppe zwar nicht mit der gängigen Definition von »Gewaltkriminalität « durch die PKS überein, überschneidet sich aber durchaus im Kernbereich. Ein weiterer erheblicher Unterschied zu der Zählweise der PKS besteht darin, dass der betroffene Täterkreis die Erheblichkeitsgrenze einer Verurteilung zu mindestens 2 Jahren Freiheitsstrafe überschrittenen haben muss.
Das derzeitige gesetzliche System im Umgang mit der genannten Tätergruppe beinhaltet als zentrale Voraussetzung das Merkmal »gefährlich«. $\mathrm{Zu}$ jeder Zeit der staatlichen Kontrolle ist es möglich, eine Gefährlichkeitsprognose einzuholen: in der Hauptverhandlung zur Frage der Verhängung einer Maßregel, zwingend vorgeschrieben bei der Frage nach der Strafrestaussetzung und neuerdings bei der Frage der Anordnung der nachträglichen Sicherungsverwahrung. Damit rückt der Begriff »Gefährlichkeit« in den Mittelpunkt. Es ist also unverzichtbar klar zu stellen, was unter diesem Begriff zu verstehen ist. Müller-Dietz macht deutlich, dass eine kriminologisch wie rechtlich eindeutige und auch einhellig akzeptierte Definition der »Gefährlichkeit« oder »Gemeingefährlichkeit « nicht existiere (S. 427). Das Gesetz hingegen nennt in $\S \S 63,64$ Abs. 1, 66 Abs. 1 Nr. 3 StGB den Begriff "gefährlich « und verweist auf die Umschreibung, dass die Gefahr bestehe, dass der Täter erhebliche rechtswidrige Taten begehen werde. Damit entspringt dieser Begriff dem Maßregelrecht und wird durch $\S 454$ Abs. 2 StPO ebenfalls in die erste Spur des deutschen Sanktionensystems transportiert. Dessecker spricht sich dafür aus, die schlichte Definition Franz Exners auch heute noch zu übernehmen: Die Gefährlichkeit bezeichne die Wahrscheinlichkeit strafbedrohter Handlungen (2004, S. 181). Dabei ist es ihm wichtig zu unterstreichen, dass die Gefährlichkeitsprognose nur deliktspezifisch und im Hinblick auf die Verhältnismäßigkeit der verhängten Maßregel zu bestimmen ist.

\section{Die Rolle der Gefährlichkeitsprognose}

Damit wird bereits das Problem des Vorschriftenkanons deutlich: In $\S 454$ Abs. 2 S. 2 StPO heißt es, dass das Gutachten sich zu der Frage zu äußern hat, ob bei dem Verurteilten keine Gefahr mehr besteht, dass dessen durch die Tat zutage getretene Gefährlichkeit fortbesteht. In den Hauptverhandlungen wird aber meist über die Gefährlichkeit nicht gesprochen. Heißt das nicht zwingend, dass nur in den Fällen, in denen auch die Gefährlichkeit in der Hauptverhandlung positiv festgestellt wurde, die genannte Vorschrift überhaupt Anwendung findet? Die Gefährlichkeit der Tätergruppe wird jedoch in $\S 454$ StPO unterstellt. Die Verhängung der Sicherungsverwahrung nach $\S 66$ Abs. 3 StGB hingegen arbeitet zu recht nicht mit Hypothesen, sondern verlangt nach § 66 Abs. 1 Nr. 3 StGB eine Gesamtwürdigung des Täters, aufgrund derer die Gefährlichkeit des Täters erst festgestellt werden muss. Das Verhältnis der Vorschriften ist deshalb zumindest im Hinblick auf die Anforderungen an die Feststellung der Gefährlichkeit unklar.

Das Urteil über das Vorliegen der Gefährlichkeit ist ein Wahrscheinlichkeitsurteil, also eine Prognose (dazu Müller-Dietz mwN). Neben den genannten Vorschriften setzen die Maßregeln der Unterbringung in einem psychiatrischen 
Krankenhaus und in einer Entziehungsanstalt gem. §§ 63, 64 StGB ebenfalls eine Gefährlichkeitsprognose voraus. Für die Anordnung der freiheitsentziehenden Maßregeln betont $\mathrm{Ha}$ nack, dass eine Zweckbetrachtung wohl mit Eindeutigkeit ergebe, dass jedenfalls bei der Frage der »Gefährlichkeit für die Allgemeinheit« und der Frage nach der "Gesamtwürdigung « verschiedenartige Anforderungen nicht bestünden (LK Vor §§ 61 ff Rn. 41). Der Anknüpfungspunkt für die Gefährlichkeit ist einerseits der Hang des Täters, Straftaten zu begehen, andererseits der psychische Defekt. Dabei ist festzustellen, dass die Auslegung dieser weiteren Voraussetzungen keine eigenständigen Konturen erlangt hat (Kinzig, S. 76). Aus diesem Grund geht Dessecker soweit zu sagen, dass das Konzept der Gefährlichkeit die einzige materielle Voraussetzung der Anordnung der freiheitsentziehenden Maßregeln sein sollte (2004, S. 302 f.).

Da die Anordnung der Sicherungsverwahrung bei der hier betrachteten Personengruppe fakultativ ausgestaltet ist, muss der Gesetzgeber folglich von einer Quantifizierbarkeit der »Gefährlichkeit« ausgegangen sein (diese Frage stellten sich bereits Eisenberg/Hackethal, S. 199). Ist der Täter extrem gefährlich, soll er gem. § 66 Abs. 3 StGB in Sicherungsverwahrung genommen werden, ist er lediglich einfach gefährlich, so bekommt er eine Freiheitsstrafe mit Begutachtungszwang bei der Strafrestaussetzung nach $\S 454$ Abs. 2 StPO. Beide Vorschriften wurden zusammen im Jahre 1998 in das Strafrecht eingefügt. Dabei ging der Gesetzgeber davon aus, dass $§ 66$ Abs. 3 StGB den Hangtäter betreffe, bei $\S 454$ Abs. 2 StPO wollte er dem erhöhten Sicherungsbedürfnis der Allgemeinheit vor besonders rückfallgefährdeten Tätern Rechnung tragen (BT-Drs. 13/7163).

Zur Zeit der Neuformulierung der Voraussetzungen der Strafrestaussetzung in §§ 57f StGB und § 454 StPO im Jahr 1998 war ausführlich diskutiert worden, ob es sich bei den Änderungen um eine Klarstellung oder um eine Verschärfung des Gesetzes handelte (vgl. statt vieler Cornel, S. 426 ff.). Es bestand weitgehend Einigkeit darüber, dass lediglich eine Klarstellung über die Abwägungskriterien bei der Aussetzungsentscheidung erfolgt sei. Aufgrund des prozessualen Erfordernisses eines Gutachters bei bestimmten Verurteilten bestand jedoch die Besorgnis, dass es sich in praktischer Hinsicht doch als eine Verschärfung darstellen könnte. Mit einer eigenen Untersuchung, basierend auf der Auswertung von Vollzugsstatistiken, Interviews und Aktenanalysen hat Cornel herausgefunden, dass »weder von einem Rückgang der Strafrestaussetzungen noch von wesentlichen, durch die Gutachtenerstellung verursachten Verzögerungen der bedingten Entlassungen gesprochen werden kann. (...) Eine Verschärfung der Strafrestaussetzungspraxis bei Sexualdelikten kann zwar nicht ausgeschlossen werden, verlässliche Anhaltspunkte dafür gab es aber nicht« (S. 438). Dieses Ergebnis gilt für den Strafvollzug. Für den Maßregelvollzug, bei dem $\S 454$ Abs. 2 StPO über $\S 463$ Abs. 3 S. 3 StPO ebenfalls zur Anwendung kommt, verzeichnet Koller hingegen in einer regionalen Analyse eine Halbierung der Entlassungszahlen für den gleichen Zeitraum (S. 3, Loccum-Manuskript). Es ist weiter zu beachten, dass Cornel alle Strafrestaussetzungen betrachtet und nicht ausdrücklich nur die hier gewählte Gruppe der in $\S 66$ Abs. 3 StGB genannten Personen untersucht.

Durch diese Auslegung der Vorschriften wird deutlich, dass der Prognoseerstellung durch den Sachverständigen erhebliches Gewicht zukommt. Gerade in Anbetracht, dass Cornel (S . 435) verzeichnet, dass die Gutachten im Vollstreckungsverfahren nur sehr selten mündlich vorgetragen werden, da in aller Regel alle Beteiligten darauf verzichten, ist die angemessene Stellung für die Gefährlichkeitsprüfung nicht das Vollstreckungsverfahren, sondern muss die Hauptverhandlung sein.

\section{Plädoyer für eine Gefährlichkeits- prüfung in der Hauptverhandlung}

Die Forderung, das Vollstreckungsverfahren schon in der Hauptverhandlung unmittelbar vorzubereiten und die notwendigen Maßnahmen unter Aufsicht des erkennenden Gerichts einzuleiten, wird seit längerer Zeit von Boetticher erhoben $(1998,2004)$. Unterstützung erhält er sowohl von Kollegen als auch aus der Wissenschaft (vgl Böhm, StraFo 2005, S. 305, die Vertreter der entsprechenden Arbeitsgruppe beim BGH und die abgedruckten Thesen von Frommel). Es ist also angebracht, die entscheidenden Argumente zu benennen, die für eine regelmäßige Gefährlichkeitsprüfung bei der genannten Tätergruppe schon in der Hauptverhandlung sprechen.

\section{In der Hauptverhandlung kann ein Reso- zialisierungskonzept entworfen werden.}

In $\S 46$ Abs. 2 S. 1 StGB heißt es, dass das Gericht Umstände abzuwägen hat, die für und gegen den Täter sprechen. Die Gefährlichkeit des Täters ist ebenfalls Grundlage dieser Bewertung. Steht eine Straferwartung von mehr als 2 Jahren im Raum, so ist bei der Sanktionenwahl neben generalpräventiven erst recht aus spezialpräventiven Gründen auch über die (Wieder-) Eingliederung des Täters in die Gesellschaft nachzudenken und nicht nur über die Frage, ob "Sicherungsverwahrung ja oder nein «. Gerade bei einschlägigen Sexualstraftätern enthält das Sanktionen- und Strafvollzugsrecht die Möglichkeiten der Durchführung einer therapeutischen Behandlung. § 9 Abs. 1 StVollzG sieht eine obligatorische Verlegung dieser Personen in die sozialtherapeutische Anstalt vor (zu dem Anspruch auf Behandlung s. Böhm StraFo 2005,
S. 184 ff.); §§ 57 Abs. 3 iVm 56c, 68b StGB eröffnen auch in den übrigen Fällen die Möglichkeit einer ambulanten therapeutischen Anschlussbetreuung nach Haftentlassung. Ist das erkennende Gericht zu der Erkenntnis gelangt, dass es sich um einen Täter der oben genannten Art handelt, so sollte ein Sachverständiger nicht nur eine Schuldfähigkeitsbeurteilung abgeben, sondern auch unabhängig von § 246a StPO zur Frage Stellung nehmen können, ob zum einen eine psychiatrische oder psychotherapeutische Behandlung des Straftäters überhaupt in Betracht kommt und zum anderen wie ein individuelles Behandlungskonzept auszusehen hätte (Boetticher 2004, S. 24). Um sich nicht erstmals unter erschwerten Bedingungen in der Justizvollzugsanstalt mit dieser Frage zu befassen, sollten diese Gesichtspunkte bei Strafantritt geklärt sein. Eine Einbeziehung des Gutachters könnte sich an dem Fragenkatalog der Göttinger Strafvollstreckungskammer an einen psychiatrischen Sachverständigen (abgedruckt in NK 4/2004, S. 153) orientieren. Damit würde gewährleistet, dass Therapieangebote im Strafvollzug aufgewertet werden und der Täter schon ab dem Beginn der Strafvollstreckung mit der Teilnahme an diesen Angeboten konfrontiert wird.

2. Das Damoklesschwert der nachträglichen Sicherungsverwahrung wird wieder ein wenig höher gehängt und gesichert.

Indem das $G$ ericht über die Gefährlichkeit schon verhandelt und die Verhängung der Sicherungsverwahrung nicht für nötig befunden hat, also eine positive Rückfallprognose festgestellt wurde, ist von einer erhöhten Therapiegeeigtheit der Probanden auszugehen. In dem umgekehrten Fall, es wird von einer Gefährlichkeit ausgegangen, aber aufgrund einer guten Therapierbarkeit keine Sicherungsverwahrung verhängt, ist die Hürde schon abgearbeitet und diese Barriere steht einer Arbeit mit dem Täter zumindest nicht entgegen. Zwar sagt die Gesetzesbegründung zur Einführung der nachträglichen Sicherungsverwahrung ausdrücklich, dass die nachträgliche Anordnung der Sicherungsverwahrung nicht von einer ablehnenden Entscheidung in demselben Verfahren ausgeschlossen werde (BT-Drs. 15/2887, S. 12). Sie wird aber durch die aufgezeigte Handhabung der Gefährlichkeit tatsächlich nur noch für wenige Einzelfälle in Frage kommen. Zwar ist die soziale Kontrolle der Gewalttäter wegen der Prüfung der Gefährlichkeit in der Hauptverhandlung erheblich erhöht: Stehen sie dann doch stets mit der Fußspitze in der Nähe der Sicherungsverwahrung. Es ist aber zu bedenken, dass auch eine Sicherungsverwahrung grundsätzlich nicht unendlich ist. Es ist in $\S 67 \mathrm{~d}$ Abs. 3 StGB eine Regelerledigung nach zehn Jahren vorgesehen. In einem maximalen Zweijahrestakt wird zudem zur währenden Gefährlichkeit des Täters Stellung genommen. Schließlich ist es ein (kriminalpolitisch) gewünschtes 
Ergebnis, dass Straftäter, deren Rückfall zur Begehung von sensiblen Straftaten wahrscheinlich ist, nicht in Freiheit gelassen werden. Boetticher macht ebenfalls auf das Problem aufmerksam, dass aufgrund taktischen Verteidigungsverhaltens der Angeklagte versuchen wird, entweder die Aussage zu verweigern oder nur Angaben zu machen, die seine Schuldunfähigkeit positiv beeinflussen ohne den Grad der Maßregelanordnungsvoraussetzungen zu erreichen. Er stellt deshalb die Überlegung an, dass die Strafvollstreckungskammern unmittelbar nach Rechtskraft des Urteils in die Einleitung und Überwachung der Behandlungsmaßnahmen einzubeziehen seien und befreit von dem Druck des Strafverfahrens mit Hilfe des bereits bestellten Gutachters eine umfassende Persönlichkeitsdiagnostik zu erstellen hätten (Boetticher 1998, S. 361). Dies setzt allerdings eine Aufteilung der Verfahrenstadien voraus, die das deutsche Gesetz so (noch) nicht kennt.

\section{Die Entlassungsprognose wird erleichtert.}

Eine Entlassungsprognose nach $\S 454$ Abs. 2 StPO kann auf der Grundlage der Feststellungen in der Hauptverhandlung positiv festgestellt werden, wenn die Prognose in der Hauptverhandlung positiv war und sich während des Vollzugs keine für die Gefährlichkeit des Täters sprechenden Umstände gezeigt haben. Aus diesem Grund bedarf es einer besonderen Rechtfertigung, wenn diesen Inhaftierten die Vollzugslockerungen versagt werden sollen. Damit werden die oben von Nedopil genannten Schwierigkeiten für die Sachverständigen erheblich gemildert und eine Strafrestaussetzung kann die Regel bleiben und entwickelt sich nicht zur Ausnahmeerscheinung. Damit wird letztendlich auch der angespannten Belegungssituation der Justizvollzugsanstalten Rechnung getragen. Auf diese Weise kommt der engagierten ambulanten Nachsorge der Straftäter begleitet durch Bewährungshilfe oder Führungsaufsicht ebenfalls die Bedeutung zu, die sie verdient. Boetticher betont, dass nach allen bisherigen Erfahrungen über Rückfälle von Straftätern allgemein und von Sexualstraftätern insbesondere die Phase der ersten Zeit nach der Entlassung besonders kritisch ist (2004, S. 50). Um erheblichen Rückfällen, die die Kriminalitätsfurcht der Bevölkerung exponential zunehmen lassen, vorzubeugen, sollte die Anschlussbetreuung des Täters in dem Vollstreckungsverfahren zum Hauptthema gemacht werden. Eine beeindruckende Zahl von bundesweiten Projekten wird bei Egg vorgestellt. Es hat sich dort gezeigt, dass die Rückfälligkeit der Straftäter erheblich reduziert werden konnte: Boetticher präsentiert für das Projekt der ambulanten Nachsorge durch die Bewährungshilfe Stuttgart e.V. bei 243 Klienten in 4 Jahren nur in zehn Fällen einen Rückfall in dieser Zeit. Freese betont ebenfalls, dass die Behandlungserfolge dort am höchsten seien, wo kriminaltherapeutische Interventionen schwerpunktmäßig ambu- lant und nicht stationär angesiedelt sind (S. 53). Er bezieht sich auf Studien zur Effizienz von Straftäterbehandlungsprogrammen, die im Mittel eine Reduktion der Rückfallraten um $12 \%$ aufwiesen, die erfolgreichsten Programme sogar eine Reduktion bis zu $40 \%$.

\section{Fazit}

Verfolgt man den vorgeschlagenen Weg, nämlich die Frage nach der konkreten Gefährlichkeit des in $\S 66$ Abs. 3 StGB beschriebenen Straftäters bereits in der Hauptverhandlung zu beantworten, so wird der Fokus wieder auf die Eingliederung des Straftäters mit entsprechender Anschlussbetreuung gelenkt. Der Aspekt der Sicherung wird zum Thema in der Hauptverhandlung. Die Gerichte haben nicht mehr die Möglichkeit, ihre Verantwortung an die Vollstreckungsorgane abzugeben. Der Vollzug kann sich folglich wieder seinem eigentlichen Ziel, der Resozialisierung der Straftäter, widmen.

\section{Literatur:}

Baltzer, Ulrich. Die Sicherung des gefährlichen Gewalttäters - eine Herausforderung an den Gesetzgeber - . Wiesbaden 2005.

Bennefeld-Kersten, Katharina: Psychisch auffällige Menschen im Gefängnis - eine Erhebung im niedersächsischen Strafvollzug. In: BewHi 2005, S. 30-40.

Boetticher, Axel/Nedopil, Norbert/Bosinski, Hartmut A. G./Saß, Henning: Mindestanforderungen für Schuldfähigkeitsgutachten. In: NStZ 2005, S. 57-62.

Boetticher, Axel: Rechtliche Rahmenbedingungen. In: Egg, Rudolf (Hrsg.): Ambulante Nachsorge nach Straf- und Maßregelvollzug. Wiesbaden 2004, S. 15-54.

Boetticher, Axel: Der neue Umgang mit Sexualstraftätern - eine Zwischenbilanz. In: MschrKrim 1998, S. 354-367.

Böhm, Klaus Michael: Der Anspruch auf Behandlung gefährlicher Gewalt- und Sexualstraftäter im Strafvollzug. In: StraFo 2005, S. 184-189.

Böhm, Klaus Michael: Anmerkung zu BGH, Urt. v. 11.5.2005. In: StraFo 2005, S. $304 \mathrm{f}$.

Cornel, Heinz: Klarstellung oder Verschärfung der Bedingungen zur Strafrestaussetzung zur Bewährung. In: MschrKrim 2002, S. 424-438.

Dessecker, Axel: Die Überlastung des Maßregelvollzugs: Folge von Verschärfungen im Kriminalrecht? In: NK 1/2005, S. 23-28.

Dessecker, Axel: Gefährlichkeit und Verhältnismäßigkeit. Berlin 2004.

Dünkel, Frieder; Geng, Bernd: Fakten zur Überbelegung im Strafvollzug und Wege zur Reduzierung von Gefangenenraten. In: NK 4/2003, S. 146-149.

Egg, Rudolf (Hrsg.): Ambulante Nachsorge nach Straf- und Maßregelvollzug. Wiesbaden 2004.

Eisenberg, Ulrich/Hackethal, Achim: »Gesetz zur Bekämpfung von Sexualdelikten und anderen gefährlichen Straftaten " vom 26.1.1998. In: ZfstrVo 1998, S. 196-202.

Elz, Jutta: Legalbewährung und kriminelle Karrieren von Sexualstraftätern. - Sexuelle Mißbrauchsdelikte -. Wiesbaden 2001.

Freese, Roland: Ambulante Versorgung von psychisch kranken Straftätern im Maßregel- und
Justizvollzug - Analysen, Entwicklungen, Impulse. In: R\&P 2003, S. 52-57.

Frommel, Monika: Begutachtung und Strafverfahren. Die Interessenlagen im Strafprozess. Manuskript der Tagung »Forensische Psychiatrie und Maßregelvollzug - Gesellschaftlicher Auftrag«, Loccum, 17.-19. Mai 2004. Demnächst abzurufen unter www.loccum.de.

Hanack, Ernst-Walter: Leipziger Kommentar Vor § 61, §§ 61-67, 11. Auflage, Berlin 1992.

Jehle, Jörg-Martin: Strafrechtliche Unterbringung in einem psychiatrischen Krankenhaus Rechtswirklichkeit und aktuelle Probleme In: BewHi 2005, S. 3-14.

Kinzig, Jörg: Die Sicherungsverwahrung auf dem Prüfstand. Freiburg i. Br. 1996.

Koller, Matthias: Begutachtung und Prognostik. Sicht der Strafvollstreckungskammer. Manuskript der Tagung »Forensische Psychiatrie und Maßregelvollzug - Gesellschaftlicher Auftrag «, Loccum, 17.-19. Mai 2004. Demnächst abzurufen unter www.loccum.de.

Müller-Dietz, Heinz: Zur Gefährlichkeitsbeurteilung bei schwerer Gewaltkriminalität. In: Festschrift Jesionek 2002, S. 423-433.

Nedopil, Norbert: Prognosebegutachtungen bei zeitlich begrenzten Frieheitsstrafen - Eine sinnvolle Lösung für problematische Fragestellungen? In: NStZ 2002, S. 344-349.

Neubacher, Frank: Die Einholung eines Sachverständigengutachtens bei der Entscheidung über die Aussetzung des Strafrestes gemäß § 57 I StGB, § 454 II StPO. In: NStZ 2001, S. 449-454.

Royen, Georg: Die Unterbringung in einem psychiatrischen Krankenhaus bzw. in einer Entziehungsanstalt nach $§ \S 63,64$ StGB als kleine Sicherungsverwahrung? In: StV 2005, S. 411-416. Speiermann, Joachim: Zur Reststrafenaussetzung. Geschichtliche Entwicklung und gegenwärtige Rechtsprechung. Konstanz 1995.

Mitglieder der an forensisch-psychiatrischen Fragen besonders interessierten interdisziplinären Arbeitsgruppe aus Juristen, forensischen Psychiatern und Psychologen sowie Sexualmedizinern:

VRinBGH Dr. Rissing-van Saan, VRiBGH Nack, RiBGH Basdorf, RiBGH Dr. Bode, RiBGH Dr. Boetticher, RiBGH Dr. Detter, RiBGH Maatz, RiBGH Pfister, VRiBGH a.D. Dr. Schäfer, die Bundesanwälte Hannich und Altvater, der Kriminologe Prof. Dr. Schöch (München), der Rechtsanwalt Dr. Deckers (Düsseldorf), die forensischen Psychiater Prof. Dr. Berner (Hamburg), Prof. Dr. Dittmann (Basel), Prof. Dr. Foerster (Tübingen), Prof. Dr. Kröber (Berlin). Prof. Dr. Leygraf (Essen), Dr. Müller-Isberner (Gießen), Prof. Dr. Nedopil (München), Prof. Dr. Saß (Aachen), Dr. Habermeyer (Rostock), die Sexualmediziner Prof. Dr. Dr. Beier (Berlin), Prof. Dr. Bosinski (Kiel) und der Rechtspsychologe Prof. Dr. Köhnken (Kiel)

Zitiert aus NStZ 2005, S. 57 